\title{
Comparing normative influences as determinants of environmentally conscious behaviours between the USA and Japan
}

\author{
Kaori Ando, ${ }^{1}$ Susumu Ohnuma ${ }^{2}$ and Edward C. Chang ${ }^{3}$ \\ ${ }^{1}$ Nara Women's University, Nara, ${ }^{2}$ Hokkaido University, Hokkaido, Japan; and ${ }^{3}$ University of Michigan, Ann Arbor, \\ Michigan, USA
}

\begin{abstract}
The present study explored the influences of subjective and descriptive norms on environmentally conscious behaviours between the USA and Japan. It was predicted that subjective norms would have a larger effect on behaviour in Japan than in the USA. Descriptive norms were expected to have a greater influence on behaviour in the USA. The survey was done with 160 American students and 114 Japanese students. The results showed that subjective norms are relevant only in Japan, but the effect was limited. Alternatively, descriptive norms were a powerful determinant in the USA. It was also found that respondents asserted that they commit environmentally conscious behaviours more frequently than others, regardless of their country. The present research suggests that the role of subjective and descriptive norms may vary between cultures, and highlights a necessity for distinguishing between interpersonal and social norms.
\end{abstract}

Key words: descriptive norm, environmentally conscious behaviour, Japan, subjective norm, USA.

\section{Introduction}

Today, environmental problems such as global warming are a common concern in the world. However, perceptions and behaviour concerning the environment are likely to differ between countries. The present study explores the determinants of environmentally conscious behaviours between the USA and Japan. The USA discharges about one-quarter of the carbon dioxide in the world, and electricity consumption per person is about two times that of Japan (OECD, 1997). While there are many discussions based on the macroscopic data, studies that investigate individual differences in environmentally conscious behaviours from a psychological perspective are few and far between.

\section{Normative influences}

The present study compares normative influences on behaviour between the USA and Japan. Subjective norms in the theory of reasoned action (TRA; Fishbein \& Ajzen, 1975; Ajzen \& Fishbein, 1980) are defined as the perceived expectations from significant others and compliance with them. The TRA has been widely applied in studying various behaviours in many countries (for review, see Terry, Gallois, \& McCamish, 1993; Blue, 1995; Albarracin, Johnson, Fishbein, \& Muellerleile, 2001; Hagger, Chatzisarantis, \& Biddle, 2002). The TRA proposes that behaviours

Correspondence: Kaori Ando, Department of Human Life and Environment, Nara Women's University, Nara 630-8506, Japan. Email: kaori@cc.nara-wu.ac.jp

Received 22 December 2004; accepted 7 February 2007. are determined by a combination of attitudes toward the behaviour and subjective norms.

It is also necessary to consider norms from a broader social context because subjective norms involve interpersonal influences only from very close others. Cialdini, Reno, and Kallgren (1990) argued that researchers may also look at other types of norms, including descriptive norms (what most others do). Revis and Sheeran, 2003) conducted meta-analysis for 21 studies which examined descriptive norms in the TRA model, and found that there was a medium to strong correlation between descriptive norms and intentions. Accordingly, their findings suggest the importance of considering descriptive norms within the TRA.

In the present study, participants' estimation of others' behaviours in their country was used to assess for descriptive norms. Kitaori and Yoshida (2004) observed illegal crossing in an intersection in Japan. These researchers found that the number of surrounding people who were illegally crossing was a strong predictor of the behaviour. Accordingly, Kitaori and Yoshida's findings indicate that the mere number of others who engage in the behaviour has effects through descriptive norms. Descriptive norms are presumed to be important especially as a determinant of environmentally conscious behaviours because environmental problems are related to social benefits: If nobody engages in such behaviours, the whole society would suffer from a damaged environment in the near future.

The present study also measured eco-net for exploratory reasons, which is the number of friends who are environmentally minded. Ando and Hirose (1999) found that the existence of environmentally minded friends had effects on participation in an environmental movement. Thus, we 
examined whether the source of influence is limited to very close others or whether environmentally minded friends who are not important in people's daily lives also have an effect on one's behaviour. The eco-net was measured in order to check whether it is only significant others, broader interpersonal networks, or norms in the whole country which may affect the individual's behaviour in Japan and in the USA.

\section{Cross-cultural differences}

Regarding cross-cultural differences in relation to subjective norms, Markus and Kitayama's (1991) view of the independent and interdependent self suggests that subjective norms may play a bigger role in Japan. They argued that views of the self vary among different cultures. For 'independent' cultures like in the USA or in Northern Europe, the self is defined as being independent of others, and the boundary of the self is clear; the aim of individuals in these cultures is to be unique from others. For 'interdependent' cultures like those found in East Asian countries including Japan, the self is defined in relation to others, and the aim in these cultures is to keep harmony with others. Therefore, in order not to offend others' feelings, people in interdependent cultures are often more aware of others' expectations.

Ajzen (1991) conducted a meta-analysis of 16 studies which used the theory of planned behaviour and found that the effect of subjective norms was not clear when compared to the effect of attitudes. However, the studies used in the meta-analysis were mostly those conducted in Western countries. Abrams, Ando, and Hinkle (1998) found that subjective norms played an important role in determining one's intention of turnover in Japan, while it did not have a significant effect in the UK. Their study suggests that the influence of subjective norms is stronger in Japan where interpersonal relationships are more important. In addition, environmental studies conducted in Japan have also shown that expectation from others played an important role (Nonami, Sugiura, Ohnuma, Yamakawa, \& Hirose, 1997; Ando \& Hirose, 1999). Overall, these findings support Markus and Kitayama's (1991) view that individuals from East Asian cultures pay more attention to their significant others' expectations and try to comply with them as much as possible.

While subjective norms represent the influence from significant others, and depends on interpersonal relationships, social norms need to be understood in intra- and intergroup contexts. Social identity theory (Tajfel \& Turner, 1986) suggests that belonging to a group constitutes a part of one's identity, which affects a number of one's cognitive and behavioural processes. From within this theory, it can be predicted that one is likely to conform to group norms when group membership is salient.

By re-examining social identity theory and collectivism, Yuki (2003) proposed that in East Asian cultures as found in Japan, people hold a strong tendency to think about groups as predominantly relationship based. In contrast, people from Western cultures such as the USA have a tendency to emphasize categorical distinctions between ingroups and outgroups. This idea of a relationship-focused group orientation for Japanese is consistent with Markus and Kitayama's (1991) notion of interdependent self and with Hamaguchi's (1977) idea of 'contextualism' (kanjin-syugi), which places importance on the relationship between individuals. In East Asian cultures, each group member is perceived as a meaningful social unit. In Western cultures, category-based group representations are more dominant and ingroup members are perceived as more homogeneous. Accordingly, processes consistent with social identity theory are expected to be most applicable to Western cultures (Yuki, Maddux, Brewer, \& Takemura, 2005). For example, Yuki et al. (2005) showed that Americans trusted strangers based on whether they shared category membership, while trust by Japanese depended more on the likelihood of sharing direct or indirect relationship links.

As category-based group representations are more dominant in Western countries (Yuki, 2003; Yuki et al., 2005), we hypothesized that estimations of others' behaviours in the country (i.e. descriptive norms within the ingroup) would play a bigger role in the USA than in Japan. However, we hypothesized that subjective norms would play a bigger role in Japan than in the USA where interpersonal relationships are more valued.

\section{The aim of the study}

The present study explores the determinants of environmentally conscious behaviour in the USA and Japan. There are few studies which have compared the determinants of environmentally conscious behaviours between the USA and Japan, although it is obvious that there are differences in behaviours at the macro level. We also aim to compare the determinants of different types of environmentally conscious behaviours because it is assumed that determinants would differ across different behavioural domains. For example, subjective norms might have stronger effects on behaviours which can be observed by others, such as shopping behaviours and recycling behaviours.

The particular objective of this study is to compare the effects of various normative influences between the USA and Japan. To assess for interpersonal norms, subjective norms were measured. As a measurement of descriptive norms, estimation of others' behaviours was used. The 
eco-net was measured to examine the influence from one's interpersonal network which is broader than subjective norms. We hypothesize that Japanese are more likely to be influenced by significant others than Americans because it is of greater importance in Japan to maintain harmonious relationships with close others. The estimation of others' behaviours would have a bigger influence in the USA because category-based group processes would be more applicable in Western cultures. We did not have any specific predictions for the eco-net because it was introduced for exploratory reasons.

\section{Method}

\section{Design and sample}

The survey was conducted on university students in the USA and Japan. In the USA, questionnaires were distributed at two large public universities, one located in the Midwest and one located in the West Coast. A total of 160 questionnaires (87 men \& 73 women) were obtained. In Japan, the survey was conducted at two national universities. A total of 114 questionnaires (45 men and 69 women) were obtained. The participants were not informed that the research project involved cross-cultural comparisons.

\section{Measures}

Scales in the questionnaire referred to environmentally conscious behaviours, estimation of others' behaviours, attitudes toward environmental issues, subjective norms, and personal networks. Scores for each scale were the mean response to the relevant items. Every question was first constructed in Japanese, then translated into English and verified by back-translation.

Environmentally conscious behaviour. Ten items measured daily environmentally conscious behaviours. Respondents were asked how often they engage in the relevant behaviour by using a four-point scale ranging from 1 (not do at all) to 4 (always do). The behaviour domains included energy conservation, water conservation, recycling, shopping, and reuse. A higher score on this scale indicates a higher frequency to engage in environmentally conscious behaviours.

Estimation of others' behaviours. We asked respondents to estimate how many people in their country (all over the USA/Japan) engage in each of the 10 environmentally conscious types of behaviour. These 10 types of behaviour corresponded to those in the behaviour scale. Responses were coded using a three-point scale (1: a few, 2: medium, 3: a lot).

Attitudes toward environmental issues. Attitudes toward environmental issues were measured by three questions using a five-point scale ranging from 1 (strongly disagree) to 5 (strongly agree). Respondents were asked whether or not they believed it is good to engage in environmentally conscious behaviours. Sample questions included: 'Is it good to engage in environmentally conscious behaviour?', and 'Is it good for society to have an environmentally conscious lifestyle?'. A higher score on this scale indicates higher pro-environmental attitudes.

Subjective norm. Subjective norm was measured by four items asking how much each of the following people would expect respondents to engage in environmentally conscious behaviours: parents, best friends, boyfriend/girlfriend, and the majority of other friends. Responses were coded using a five-point scale. A higher score indicates a higher expectation.

Personal network. The number of friends who are environmentally minded was measured. This scale is named an 'eco-net'. The eco-net was measured by seven questions, asking how many environmentally minded friends respondents have. Sample questions were: 'Your friend(s) who are interested in environmental issues', 'Your friend(s) who are well acquainted with environmental issues', and 'Your friend(s) who are members of an environmental group'. The responses in the measures of the eco-net were all transformed into logarithm first and then mean scores were calculated because of the great variations in responses.

\section{Results}

\section{Demographics}

The average age of the American sample was 20.8 years old, ranging from 18 to 34 years of age, while that of the Japanese sample was 20.6, ranging from 18 to 46 years of age. There was no significant difference in age between the samples. In Japan, $51 \%$ of the students lived by themselves, whereas the corresponding number was only $14 \%$ in the USA, which indicates that many students share houses or flats with friends in the USA $\left(\chi^{2}(4)=86.0, p<0.001\right)$.

\section{Scale construction}

At first, a factor analysis with Varimax rotation was conducted on the behavioural measures (Table 1). Three factors emerged. The first factor was interpreted as 'resource conservation behaviour'. Statements such as 'turn off faucets when I shampoo my hair' or 'take my own bag for shopping' weighed heavily on this factor. The second factor included the statements; 'turn off lights when you leave rooms', and 'switch off air conditioners frequently'. This factor was named 'energy conservation behaviour'. 
Table 1 Factor loadings of behavioural measures

\begin{tabular}{lccc}
\hline & Factor 1 & Factor 2 & Factor 3 \\
\hline Turn off faucets when I shampoo my hair & 0.77 & \\
Prefer products with a green mark & 0.66 & \\
Take own bag for shopping & 0.66 & \\
Turn off faucets when I brush my teeth & 0.56 & 0.78 \\
Turn off the lights & & 0.77 & 0.67 \\
Switch off air conditioner frequently & & & 0.80 \\
Keep the temperature moderately & & & 0.77 \\
Take old items to second hand shops & 0.42 & 1.88 & 0.44 \\
Take old books to second hand shops & 2.05 & 1.59
\end{tabular}

Table 2 Mean scores and standard deviations of each type of environmentally conscious behaviour

\begin{tabular}{|c|c|c|c|c|c|c|c|}
\hline \multirow[b]{2}{*}{ Country } & & \multicolumn{3}{|c|}{ Own behaviour } & \multicolumn{3}{|c|}{ Estimation of others' behaviours } \\
\hline & & $\begin{array}{l}\text { Water conservation } \\
\text { and shopping }\end{array}$ & $\begin{array}{c}\text { Energy } \\
\text { conversation }\end{array}$ & Re-use & $\begin{array}{l}\text { Water conservation } \\
\text { and shopping }\end{array}$ & $\begin{array}{c}\text { Energy } \\
\text { conservation }\end{array}$ & Re-use \\
\hline \multirow[t]{2}{*}{ USA } & $M$ & 1.86 & 2.86 & 2.49 & 1.67 & 1.35 & 1.85 \\
\hline & $S D$ & 0.53 & 0.72 & 0.80 & 0.47 & 0.29 & 0.43 \\
\hline \multirow[t]{2}{*}{ Japan } & $M$ & 2.58 & 2.97 & 2.26 & 1.81 & 1.90 & 1.96 \\
\hline & $S D$ & 0.69 & 0.80 & 0.70 & 0.48 & 0.41 & 0.55 \\
\hline
\end{tabular}

The third factor included 'take old items to second hand shops or garage sales' and 'take old books to second hand shops'. This factor was named 're-use behaviour'.

For subsequent analyses, scores on each behaviour scale were calculated based on the means of the items which loaded heavily on each factor. Scores of the three types of behaviours for estimation of others' behaviours were calculated in the same way using the same factor structure as own behaviour.

Cronbach's alphas for the scales used in the analysis were as follows: environmentally conscious behaviours (resource conservation behaviour, 0.62; energy conservation behaviour, 0.67; re-use behaviour, 0.52), estimation of others' behaviours (resource conservation behaviour, 0.64 ; energy conservation behaviour, 0.68 ; re-use behaviour, 0.53 ), attitudes toward environmental issues $(0.84)$, subjective norm (0.76) and eco-net (0.86). The results indicated an almost satisfactory level of reliability for each of the measurements.

\section{Cultural differences of environmentally conscious behaviours}

In order to examine whether mean scores on each of the behavioural scales differed between countries, between own behaviour and estimation of others' behaviours, we ran a 2 (country: USA $v s$ Japan) $\times 2$ (agent: self $v s$ others) $\times 3$ (types of behaviour: resource conservation $v s$ energy conservation $v s$ re-use) analysis of variance (ANOVA) with repeated measures with the last two factors. Cell means and standard deviations are shown in Table 2.

First, all of the main effects were significant (country: $F_{1,265}=29.7$, agent: $F_{1,265}=474.0$, types of behaviour: $F_{2,530}=60.0$; all $\left.p s<0.01\right) . F$-value for the main effect of agent was particularly large, indicating that respondents estimated that they engaged in environmentally conscious behaviours more frequently than others in the country $(M=2.51$ vs 1.76). Behavioural level of Japanese respondents was higher than American respondents $(M=2.25 \mathrm{vs}$ 2.02 ), and re-use behaviour was conducted or estimated to be conducted more frequently than resource conservation behaviour and energy conservation behaviour $(M=2.42 \mathrm{vs}$ 1.98 vs 2.00, respectively).

We also found significant interactions between country and types of behaviour $\left(F_{2,530}=45.1, p<0.001\right)$, agent and types of behaviour $\left(F_{2,530}=29.7, p<0.001\right)$. The significant interaction effect between country, agent and types of behaviour was also observed $\left(F_{2,530}=39.8, p<0.001\right)$. As Table 2 shows, respondents estimated that they engaged in energy conservation behaviours most frequently, while 
Table 3 Zero-order correlations between variables in the USA and Japan

\begin{tabular}{lccccccccc}
\hline & 1 & 2 & 3 & 4 & 5 & 6 & 7 & 8 \\
\hline 1 Attitude & & $0.19^{*}$ & 0.11 & 0.02 & 0.08 & 0.01 & $0.40^{* * *}$ & $0.35^{* * *}$ & $0.29 * * *$ \\
2 Subjective norm & $0.27 * *$ & & $0.59 * * *$ & 0.02 & 0.05 & 0.05 & $0.16^{*}$ & 0.13 & $0.21^{* * *}$ \\
3 Eco-net & 0.04 & $0.22^{*}$ & & -0.09 & -0.03 & -0.05 & $0.25^{* *}$ & 0.09 & $0.17^{*}$ \\
4 Estimation (water) & 0.09 & 0.05 & $-0.24 *$ & & $0.34 * * *$ & $0.36^{* * *}$ & $0.26^{* * *}$ & 0.10 & 0.06 \\
5 Estimation (energy) & 0.00 & -0.05 & -0.13 & $0.44^{* * *}$ & & $0.35^{* * *}$ & 0.01 & $0.44^{* * *}$ & 0.08 \\
6 Estimation (re-use) & 0.07 & 0.15 & -0.08 & $0.41^{* * *}$ & $0.38^{* * *}$ & & 0.04 & 0.11 \\
7 Behaviour (water) & $0.22^{*}$ & $0.28^{* *}$ & 0.03 & $0.24^{* *}$ & -0.18 & -0.05 & $0.46^{* * *}$ \\
8 Behaviour (energy) & $0.22^{*}$ & $0.19^{*}$ & 0.13 & -0.03 & 0.15 & -0.01 & $0.28^{* *}$ & $0.44^{* * *}$ & $0.46^{* * *}$ \\
9 Behaviour (re-use) & 0.10 & 0.13 & 0.17 & 0.05 & 0.06 & $0.33^{* * *}$ & $0.21^{*}$ & $0.25^{* *}$ & $0.39^{* * *}$ \\
\hline
\end{tabular}

$* * * p<0.001, * * p<0.01, * p<0.05$.

Correlations for the American sample are above the diagonal; those for the Japanese sample are below the diagonal.

Estimation, estimation of others' behaviours, Behaviour, own behaviour.

others engaged in re-use behaviours most frequently. The table further indicates that the difference between self versus others was largest for energy conservation in the USA, while it was largest for resource conservation in Japan. It is also noteworthy that an interaction between country and agent was not found to be significant, indicating that respondents assumed that they conduct environmentally conscious behaviours more frequently than others regardless of their country.

\section{Cultural differences in other variables}

Using country as the dependent variable, $t$-tests were conducted for each of the main variables separately except for own behaviours and estimation of others' behaviours. There was no significant difference between the two countries for attitudes. Means of attitude were high in both countries (over 4.3 in both countries), indicating high concern for environmental problems in both countries. The Japanese students, compared to American students, scored higher on subjective norm $(M=2.82$ vs $2.48 ; t(269)=3.36$, $p<0.001$ ). Japanese students perceived higher expectations from significant others to engage in environmentally conscious behaviours. The number of eco-net was higher in the USA $(M=0.68 v s 0.22 ; t(263)=7.73, p<0.001)$. American students showed a larger network of environmentally minded friends. It may seem like a contradiction that Japanese scored higher on subjective norm but had a smaller eco-net. However, it is not so uncommon in Japanese society given that people are required to read others' expectations even if it is not spoken clearly. Moreover, subjective norm measures significant others such as family, but econet measures a broader network, including weak ties. Japanese students talk about environmental issues with family members or very close others, but do not talk with distant friends about such issues in daily life.

\section{Determinants of behaviour}

The major focus of the present study is to compare the determinants of environmentally conscious behaviour between USA and Japan. Before conducting the regression analysis, correlation coefficients between variables were examined (Table 3 ) and the results showed that a possibility of multicolineality was not high with the present data.

Scores on subjective norm and on the eco-net were found to have a stronger correlation in the USA, $r(152)=0.59$, than in Japan, $r(113)=0.22$, which indicates that in the USA, expectations from specific others were not clearly distinguished from the attitudes of various friends. Subjective norm and eco-net were not related with any kind of estimation in the USA and Japan, except for an association between eco-net and estimation of others on resource conservation behaviour in Japan. Respondents perceived that even if they are expected to engage in environmentally conscious behaviour from significant others, it did not mean everyone in the country shared the same notion.

Multiple regression analyses were conducted to explore the determinants of environmentally conscious behaviours for the USA and Japan separately. Each type of environmentally conscious behaviour was used as a dependent variable. Independent variables were attitude, subjective norm, and the corresponding estimation of others' behaviours. The results are shown in Table 4.

As the results show, attitude was a significant positive determinant of all kinds of behaviours in the USA, whereas it was not significant in any behavioural domain in Japan. Subjective norm was a significant positive determinant of resource conservation behaviour in Japan, whereas it failed to be a significant determinant in the USA for any of the behavioural domains. Eco-net was a positive determinant of resource conservation behaviour in the USA and a positive determinant of re-use behaviour in Japan. Estimation of others' behaviours was a positive significant determinant of 
Table 4 Multiple regressions for each type of behaviour

\begin{tabular}{|c|c|c|c|c|c|c|}
\hline & \multicolumn{2}{|c|}{$\begin{array}{l}\text { Water conversation } \\
\text { and shopping behaviour }\end{array}$} & \multicolumn{2}{|c|}{$\begin{array}{c}\text { Energy } \\
\text { conservation behaviour }\end{array}$} & \multicolumn{2}{|c|}{ Re-use behaviour } \\
\hline & USA & Japan & USA & Japan & USA & Japan \\
\hline Attitude & $0.36 * * *$ & 0.13 & $0.33 * * *$ & 0.19 & $0.26 * * *$ & 0.07 \\
\hline Subjective norm & -0.07 & $0.22 *$ & -0.06 & 0.13 & 0.05 & 0.03 \\
\hline Eco-net & $0.27 *$ & 0.03 & 0.09 & 0.11 & 0.13 & $0.18 *$ \\
\hline Estimation of others' behaviours & $0.25^{*}$ & $0.22 *$ & $0.39 * * *$ & 0.17 & $0.46 * * *$ & $0.33 * * *$ \\
\hline$R$-square & 0.25 & 0.14 & 0.28 & 0.11 & 0.32 & 0.15 \\
\hline$F$ value & $11.8 * * *$ & $4.4 * *$ & $13.7 * * *$ & $3.2^{*}$ & $16.3 * * *$ & $4.77 * *$ \\
\hline
\end{tabular}

$* * * p<0.001, * * p<0.01, * p<0.05$.

all types of behaviours in the USA and Japan except for energy conservation behaviour in Japan.

All of the regression models were significant, but $F$-values and $R$-square values were higher for the models involving American samples. None of the variables succeeded in explaining for significant amounts of variance in energy conservation behaviour in Japan. Accordingly, other variables not measured in the present study may be involved as determinants of this type of environmentally conscious behaviour in Japan.

\section{Discussion}

\section{Environmentally conscious behaviour}

Findings from the present study showed that many people are aware of the importance of protecting the environment, as indicated by high mean scores on attitude in both countries. However, there were some differences in the actual behaviours between the countries. The results indicated that environmentally conscious behaviours are multifactorial, and the mean scores differ depending on the behavioural domains.

In comparing own behaviours and estimation of others' behaviours, we found that respondents perceived that they commit environmentally conscious behaviours more frequently than others. This pattern was found both in the USA and Japan. Various cross-cultural studies showed that Japanese students show self-devaluating biases compared to Western students who are likely to show self-enhancing biases (Kashima \& Triandis, 1986; Heine, Takata, \& Lerman, 2000). However, the present results showed that Japanese respondents also estimated that others engaged in environmentally conscious behaviours less often than they do. The results also indicated that Japanese have selfenhancing biases regarding their behaviour. This finding is consistent with Kitayama and Karasawa's study (1997), which found that Japanese students attached positive feelings to their own selves.
The estimation of others' behaviours also differed between behavioural domains. Both American and Japanese respondents evaluated that they engaged in energy conservation behaviour most frequently, but perceived others engaged in re-use behaviour most frequently. Because people mostly engage in energy conservation behaviours at home, it is less likely that this type of behaviour is observed from outside. In contrast, re-use behaviours, such as taking old items to recycle shops, are easier to be detected by others. Thus, it may be that others' behaviours were underestimated because they were more difficult to be observed.

This tendency to underestimate others' behaviours may affect one's own behaviour because estimation of others' behaviours was found to be a strong predictor of own behaviour in the USA and in Japan. Put another way, it may be possible to increase people's engagement in environmentally conscious behaviours if they were made to believe that others also engaged in environmentally conscious behaviours frequently.

\section{Normative influences}

The regression analyses showed that the effect of subjective norm was present only for resource conservation behaviours in Japan. It did not account for any significant variance in behaviours in the USA. Findings from some previous studies (Hamaguchi, 1977; Markus \& Kitayama, 1991) have suggested that people are more aware of others' thoughts and expectations in Japan because it is more adaptable in this society. Our present findings partly support this view. They showed that the influence of a few important people like parents and close friends are of particular importance in determining resource conservation behaviour in Japan. As subjective norm was not found to be a significant predictor of energy conservation and re-use behaviours in Japan, our hypothesis was not fully supported. Nonetheless, our findings showed that the effect of subjective norm differed between cultures and between different behavioural domains. 
Estimation of others' behaviour was a powerful determinant of behaviour in the USA. It was a significant determinant of all behavioural domains and was a stronger predictor than subjective norm. Our findings also showed that in the USA, people are likely to be influenced by descriptive norms of a large category rather than expectations from very close people. Our results regarding eco-net were rather mixed. They were partly significant both in the USA and in Japan.

The results in the USA indicated that the effect of direct expectations from very close others was relatively weak, but descriptive norms had stronger effects. As Markus and Kitayama (1991) discussed, independence is valued over interdependence in the USA, and thus people would feel less pressure to conform to the significant others' expectations compared to Japanese. However, it does not mean Americans are totally unaffected by social norms. According to Yuki (2003), category-based social representations are dominant in the USA, which means one is likely to perceive the whole group to be homogeneous. The results of the present study may indicate that descriptive norms are more powerful in the USA because the notion that many people engage in the behaviour is important in a society where people are perceived to be homogeneous. It is also necessary to point out that estimation of others' behaviours was also found to be significant in Japan in two behavioural domains. It means that descriptive norms were also relevant in Japanese society, but the effect of descriptive norm appeared more clearly in the USA.

Another possible explanation for the results in the USA is the effect of false consensus, to the tendency to overestimate the percentage of others' behaviour who engage in the same behaviour as oneself. Accordingly, one might argue that the false consensus effect was stronger in the USA. One might be likely to see others as having the same attitudes as oneself in the USA. However, respondents in both countries evaluated that they engaged in energy conservation behaviours most frequently, but they perceived that others engaged in re-use behaviours most frequently. If it is the effect of false consensus, it is difficult to explain this difference in behavioural domains. It would be necessary in future research to clarify the causal relationship by experimental design or by panel study.

\section{Conclusion}

The present results give us some insights to help understand environmentally conscious behaviour in the USA and Japan. In the USA, estimation of others' behaviours was an important variable that affected one's behaviour. This suggests that if the number of people who engage in environmentally conscious behaviour becomes higher than a certain percentage, then other people would follow suit, thus increasing the overall percentage of people who would engage in such behaviours.

For future research, it would be useful to distinguish between descriptive norms and false consensus processes in analyzing the data. In the present research, it was not clear whether one engaged in environmentally conscious behaviours because others did, or just because one perceived others engaged in the same behaviours as oneself. It would also be helpful to clarify the concept of network. The econet in the present study had some conceptual overlap with descriptive norms, but results of the present study showed that the eco-net was one of the important variables that determined behaviour. Therefore, it would be interesting to further explore the role of social networks in the USA and in Japan.

Another limitation of the present study is that we only compared the USA and Japan using university students as respondents. In order to generalize the present results, it would be important to use more representative samples from other countries which perhaps have different perspectives on environmental issues.

\section{Acknowledgements}

We thank Y. Hirose for kind advice for this project. We are also thankful to K. Ohbuchi for helpful comments on this article. This study was supported by a grant from the Japanese Ministry of Education and Science (No. 14780062).

\section{References}

Abrams, D., Ando, K. \& Hinkle, S. (1998). Psychological attachment to the group: Cross-cultural differences in organizational identifications and subjective norms as predictors of workers' turnover intentions. Personality and Social Psychology Bulletin, 24, 1027-1039.

Ajzen, I. (1991). The theory of planned behaviour. Organizational Behaviour and Human Decision Processes, 50, 179-211.

Ajzen, I. \& Fishbein, M. (1980). Understanding Attitudes and Predicting Social Behaviour. Englewood Cliffs, NJ: Prentice Hall.

Albarracin, D., Johnson, B. T., Fishbein, M. \& Muellerleile, P. A. (2001). Theories of reasoned action and planned behaviour as models of condom use: A meta-analysis. Psychological Bulletin, 127, 142-161.

Ando, K. \& Hirose, Y. (1999). Kankyou volunteer dantai niokeru katsudoukeizokuito sekkyokutekisankaito no kiteiin [Determinants of turnover and willingness to exert efforts in environmental volunteer groups]. Japanese Journal of Social Psychology, 15, 90-99 (in Japanese).

Blue, C. L. (1995). The predictive capacity of the theory of reasoned action and the theory of planned behaviour in exercise research: An integrated literature review. Research in Nursing and Health, 18, 105-121. 
Cialdini, R. B., Reno, R. R. \& Kallgren, C. A. (1990). A focus theory of normative conduct: Recycling the concept of norms to reduce littering in public places. Journal of Personality and Social Psychology, 58, 1015-1026.

Fishbein, M. \& Ajzen, I. (1975). Belief, Attitude, Intention and Behaviour: an Introduction to Theory and Research. Reading, MA: Addison-Wesley.

Hagger, M. S., Chatzisarantis, N. L. D. \& Biddle, S. J. H. (2002). A meta-analytic review of the theories of reasoned action and planned behaviour in physical activity: Predictive validity and the contribution of additional variables. Journal of Sport and Exercise Psychology, 24, 3-32.

Hamaguchi, E. (1977). Nihonrashisa no saihakken [Rediscovery of Japaneseness]. Tokyo: Nippon Keizai Shinbunsha.

Heine, S. J., Takata, T. \& Lehman, D. R. (2000). Beyond selfpresentation: Evidence for self-criticism among Japanese. Personality and Social Psychology Bulletin, 26, 71-78.

Kashima, Y. \& Triandis, H. C. (1986). The self-serving bias in attributions as a coping strategy: A cross-cultural study. Journal of Cross-Cultural Psychology, 17, 83-97.

Kitaori, M. \& Yoshida, T. (2004). An observational study of the illegal crossing behaviour of pedestrians: Focusing on the effects of hurrying and habit. Japanese Journal of Social Psychology, 19, 234-240.

Kitayama, S. \& Karasawa, M. (1997). Implicit self-esteem in Japan: Name letters and birthday numbers. Personality and Social Psychology Bulletin, 23, 736-742.

Markus, H. R. \& Kitayama, S. (1991). Culture and the self: Implications for cognition, emotion, and motivation. Psychological Review, 98, 224-253.
Nonami, H., Sugiura, J., Ohnuma, S., Yamakawa, H. \& Hirose, Y. (1997). Sigen recycle koudou no ishiketteiniokeru tayouna media no yakuwari: Path kaiseki wo mothiita kentou [The role of various media in the decision making processes for recycling behaviour: A path analysis model]. The Japanese Journal of Psychology, 68, 264-271 (in Japanese).

OECD (1997). OECD Environmental Data Compendium 1997. Paris: Organization for Economic Co-operation and Development and Tokyo: Institute for Techno-Economics.

Revis, A. \& Sheeran, P. (2003). Descriptive norms as an additional predictor in the theory of planned behaviour: A meta-analysis. Current Psychology: Developmental, Learning, Personality, Social, 22, 218-233.

Tajfel, H. \& Turner, J. C. (1986). The social identity theory of intergroup relations. In: S. Worchel \& W. G. Austin, eds. Psychology of Intergroup Relations, pp. 7-24. Chicago, IL: Nelson-Hall.

Terry, D. J., Gallois, C. \& McCamish, M., eds. (1993). The Theory of Reasoned Action: Its Application to AIDS-Preventive Behaviour. Oxford: Pergamon Press.

Yuki, M. (2003). Intergroup comparison versus intragroup relationships: A cross-cultural examination of social identity theory in North American and East Asian cultural contexts. Social Psychology Quarterly, 66, 166-183.

Yuki, M., Maddux, W. W., Brewer, M. B. \& Takemura, K. (2005). Cross-cultural differences in relationship- and group-based trust. Personality and Social Psychology Bulletin, 31, 48-62. 